\title{
Image Denoising Based on the Modied ROF Model
}

\author{
Yan Jiang ${ }^{1}$ and Shu-Ling Zhang ${ }^{1}$
}

\begin{abstract}
In this paper a new image denoising model based on the ROF model is proposed. This proposed model can reduce the noise effect and also damp the staircase effect generated by the ROF model. Existence and uniqueness of solution are proved. Numerical examples illustrate advantages of our proposed model.
\end{abstract}

Keywords: Image Denoising; Euler-Lagrange Equation; Total Variation.

\section{Introduction}

Digital images, no matter what their source, usually introduce some random noise. Consequently,

a fundamental problem in image processing is the image restoration. Early methods for doing this were based on least squares and had the unfortunate property of either smoothing edges or creating spurious oscillations near edges, i.e., the well known ringing phenomenon ${ }^{[1,2]}$. But for digital images the most remarkable characteristics are edges and textures. So many researchers have been devoted efforts to the study of modified models in order to reduce this phenomenon that can eliminate noise and simultaneously preserve edges and small scale characteristics. Among the modified models, the variational models have been extremely successful in a wide variety of image restoration problems and still remain one of the most active areas of research in mathematical image processing and computer vision. The typical variational model is the ROF model ${ }^{[3]}$. Let $u$ be the original image, $f$ is the observed image and $\eta$ is the additional noise. Then the degradation image can be modeled as follows:

$\mathrm{f}=\mathrm{u}+\eta$.

Thus, the ROF model is written as follows:

$$
\min _{u \in B V(\Omega)} \frac{1}{2}\|u-f\|_{L^{2}}^{2}+\lambda|u| B V,
$$

1. Foundation department, Zhengzhou Tourism College, Zhengzhou, 45009, China

E-mail: yanjiang0628@163.com (Corresponding Author), slzhang@163.com 
where BV $(\Omega)$ denotes the space of functions with bounded variation on $\Omega$ and $|$. denotes the BV seminorm defined by

$$
|u|_{B V}=\int_{\Omega}|\nabla u| d x d y=\int_{\Omega} \sqrt{u_{x}^{2}+u_{y}^{2}} d x d y .
$$

As a result, with the norm $\|u\|_{B V}=|u|_{L^{1}}+|u|_{B V}, \mathrm{BV}(\Omega)$ is a Banach space ${ }^{[4]}$.

In the above

ROF model, if $\mathrm{k}$ is identity, the corresponding problem is called a denoising problem. In this paper we assume $\mathrm{k}$ is identity unless otherwise it is stated.

In the ROF model, $\lambda$ is a tuning parameter. Small $\lambda$ corresponds to very little noise removal, and hence $u$ is close to $f$. Large $\lambda$ yields a blurry, oversmoothed $u$. The first term in the energy functional in ${ }^{[4]}$ is the fidelity term. The second term is the regularization term to remove noise or small details. The ROF model does an excellent job in preserving edges since the diffusion with this PDE is along edges.

However, the ROF model often causes "staircase" effects since it favors solutions that are piecewise constant ${ }^{[5]}$. Staircase solutions fail to satisfy the eye and they can develop "false edges" that do not exist in the true image. To overcome this spurious limitation, in the recent decade, some higher-order PDEs have been introduced in image restoration ${ }^{[7,8,10,11]}$. Fourth-order linear or nonlinear diffusion requires much stronger smoothness and can damp oscillation much faster than second-order diffusion. On the other hand, fourth-order PDEs usually evolve an observed towards an almost smooth image and make image look more natural. But these models increase calculated costs by solving related fourthorder PDEs. Recently, Chan and Esedoglu proposed to replace the fidelity term in ROF model by $|\mathrm{u}-\mathrm{f}|_{\mathrm{L}}{ }^{1}$ in ${ }^{[9]}$. It has a good restoration but this energy function is not strictly convex, so the solution is not unique. On the other hand it has two nonsmooth terms, so it increases the calculating difficulty. In this paper we propose a modified ROF model that can effectively damp the staircase effects and have a unique solution. Simultaneously, this model do not require more calculated costs than PDEs. Then the modified model for modified ROF model(MROF) is proposed as follows:

$$
\min _{u \in B V(\Omega)} \frac{1}{2}\|u-f\|_{L^{2}}^{2}+\lambda|u| T V-\frac{\alpha}{2}\left(\|u\|_{L^{2}}^{2}+\|\nabla u\|_{L^{2}}^{2}\right),
$$

where $\lambda>0$ is the coefficient of regularity term and $\alpha>0$ is the coefficient of penalty 
function.

The plan of the paper is as follows. We fist recall some models that are mentioned in the above literature and propose our modified model. In section 3, numerical results are represented to demonstrate the effectiveness of the proposed model. Some concluding remarks are presented in last section.

\section{A Modied ROF Model for Image Restoration}

In this section we state several basic definitions and deduce some related results. We assume $\mathrm{H}$ is the Hilbert space and let $\mathrm{C}$ be a subset of $\mathrm{H}$. We also assume $\mathrm{f}_{*}$ is a minimizer of the functional $\mathrm{J}$.

Definition 2.1. $\quad \mathrm{J}: \mathrm{H} \rightarrow \mathrm{R}$ is weakly lower semicontinuous if

$$
J\left(f_{*}\right) \leq \lim _{n \rightarrow \infty} J\left(f_{n}\right),
$$

whenever $f_{n} \rightarrow f_{*}$

Definition 2.2. A functional $\mathrm{J}: \mathrm{H} \rightarrow \mathrm{R}$ is coercive if

$$
J\left(f_{n}\right) \rightarrow \infty
$$

whenever $\left\|f_{n}\right\| \rightarrow \infty$.

In [1], vogel gave the existence and uniqueness of the minimizer of $\mathrm{J}$.

Lemma 2.1. Assume that $J: H \rightarrow R$ is weakly lower semicontinuous and coercive and that $\mathrm{C}$ is a closed, convex subset of $\mathrm{H}$. Then $\mathrm{J}$ has a minimizer over $\mathrm{C}$. If $\mathrm{J}$ is also strictly convex, then the minimizer is unique. In this paper, we always assume that $\lambda>>$ $\alpha$. For notational simplicity, we denote the objective function in the modified model (1.4) as

$$
F(u)=\frac{1}{2}\|u-f\|_{L^{2}}^{2}+\lambda|u| T V-\frac{\alpha}{2}\left(\|u\|_{L^{2}}^{2}+\|\nabla u\|_{L^{2}}^{2}\right),
$$

Theorem 2.1. Assume $\mathrm{f} \in \mathrm{BV}(\Omega)$ and $\lambda \gg \alpha$. Then the minimization problem (1.4) exists a unique solution.

Proof. We notice that: if $\lambda>>\alpha$, then $F$ is weakly lower semicontinuous and coercive and $\mathrm{F}$ is finite for at least one BV image $\mathrm{u}$. Simultaneously, a sequence of images in BV $(\Omega)$ with bounded F values must be bounded as in BV $(\Omega)$ and BV $(\Omega)$ can be compactly embedded in $\mathrm{L}^{1}(\Omega)$. So, by Lemma 2.1 , it is not difficult to deduce that the 
minimizer of $F$ exists and is unique.

Theorem 2.2. If the minimization problem (1.4) satisfies Neumann adiabatic condition $\frac{\partial u}{\partial n}=0$.

along the boundary $\partial \Omega$. .Then the solution of (4) satisfies the following Euler-Lagrange equation

$(1-\alpha) u+\nabla \cdot\left(\alpha \nabla u-\frac{\lambda \nabla u}{|\nabla u|}\right)=f$.

Proof. For every $v \in C_{0}^{1}(\Omega)$, the directional derivative of $\mathrm{F}(\mathrm{u})$ is given by

$$
\begin{gathered}
\left.\frac{d}{d \varepsilon}\right|_{\varepsilon=0} F^{\prime}(u+\varepsilon v) \\
\left.\frac{d}{d \varepsilon}\right|_{\varepsilon=0}\left\{\frac{1}{2} \int_{\Omega}|u+\varepsilon v-f|^{2} d x d y+\lambda \int_{\Omega}|\nabla(u+\varepsilon v)| d x d y\right. \\
\left.\quad-\frac{\alpha}{2}\left(\int_{\Omega}|\nabla(u+\varepsilon v)|^{2} d x d y+\int_{\Omega}|u+\varepsilon v|^{2} d x d y\right)\right\} \\
=\int_{\Omega}(u-f) v d x d y+\lambda \int_{\Omega} \frac{\nabla u \nabla v}{|\nabla u|} d x d y- \\
\alpha\left(\int_{\Omega} \nabla u \nabla v d x d y+\int_{\Omega} u v\right) d x d y .
\end{gathered}
$$

Recall the Green's formulas which take the form

$$
\int_{\Omega} V \cdot \nabla v d x d y=\int_{\partial \Omega} V \cdot N v d s-\int_{\Omega} \nabla \cdot V v d x d y,
$$

where $\mathrm{N}=\left(\mathrm{n}_{1}, \mathrm{n}_{2}\right)$ is the unit outer normal vector of $\partial(\Omega)$ and $V=\frac{\nabla u}{|\nabla u|}$ and by the Neumann adiabatic condition and the variational theory, we have the related Euler-Lagrange equation as follows

$$
(1-\alpha) u+\nabla \cdot\left(\partial \nabla u-\frac{\lambda \nabla u}{|\nabla u|}\right)=f .
$$


This completes the proof.

Using the steepest descent method as did in [3], we are able to derive the associated heat flow

for our modified model (4),

$$
\frac{\partial u}{\partial t}=(1-\alpha) u+\nabla \cdot\left(\alpha \nabla u-\frac{\lambda \nabla u}{|\nabla u|}\right)-f
$$

\section{Implementation details and numerical examples}

In this section, we present some numerical results generated by our modified model and the ROF model [3]. Simultaneously, we also compared these results. We consider only twodimensional cases and restrict ourself to gray-value images, but the model can handle any dimension and can be extended to vector-valued image as well. We assume that $\Omega$ is square image region and t is the time step. Let us divide $\Omega$ into $\mathrm{n}^{2}$ subsquare domains $\Omega_{\mathrm{i}, \mathrm{j}}$. Then $\mathrm{x}_{\mathrm{i}, \mathrm{j}}=\left(\mathrm{ih}, \mathrm{jh}\right.$ ) is theendpoint of $\Omega_{\mathrm{i}, \mathrm{j}}$ for $0 \leqslant \mathrm{i}, \mathrm{j} \leqslant \mathrm{n}$, where $h=\frac{|\Omega|}{n^{2}} \quad($ here let $\mathrm{h}=1)$ and the solution is approximated by $\mathrm{u}_{\mathrm{i}, \mathrm{j}}$ at the point $\mathrm{x}_{\mathrm{i}, \mathrm{j}}$.

So we have

$$
\begin{gathered}
D_{x}^{ \pm}\left(u_{i, j}\right):= \pm\left[u_{i \pm 1, j}-u_{i, j}\right], \quad D_{y}^{ \pm}\left(u_{i, j}\right):= \pm\left[u_{i, j \pm 1}-u_{i, j}\right], \\
D_{x x}\left(u_{i, j}\right):=\left[D_{x}^{+}\left(u_{i, j}\right)-D_{x}^{+}\left(u_{i-1, j}\right)\right], \\
\left.D_{y y}(u,):=\left[D^{+}\left(u_{i j}\right)-D^{+}{ }_{x} u u_{i j-1}\right]\right]^{\prime} \\
\left|D_{x}\left(u_{i, j}\right)\right|_{\beta}=\sqrt{\left(D_{x}^{+}\left(u_{i, j}\right)\right)^{2}+\left(m\left[D_{y}^{+}\left(u_{i, j}\right), D_{y}^{-}\left(u_{i, j}\right)\right]\right)^{2}+\beta} \\
\left|D_{y}\left(u_{i, j}\right)\right|_{\beta}=\sqrt{\left(D_{y}^{+}\left(u_{i, j}\right)\right)^{2}+\left(m\left[D_{x}^{+}\left(u_{i, j}\right), D_{x}^{-}\left(u_{i, j}\right)\right]\right)^{2}+\beta}
\end{gathered}
$$

where $\mathrm{m}[\mathrm{a}, \mathrm{b}]=\left(\frac{\text { signa }+\operatorname{signb}}{2}\right) \min (|\mathrm{a}|,|\mathrm{b}|)$ and $\beta>0$ is sufficiently near to 0 in order to avoid the 
nonsmoothing term. Thus the details of the algorithm for modified model 1.4 is given as follows

$$
\begin{gathered}
u_{i, j}^{n+1}=u_{i, j}^{n}+\tau(1-\alpha) u_{i, j}^{n}+D_{x}^{-}\left(\alpha D_{x}^{+} u_{i, j}^{n}-\lambda \frac{D_{x}^{+} u_{i, j}^{n}}{\left|D_{x} u_{i, j}^{n}\right|_{\beta}}\right)+ \\
D_{y}^{-}\left(\alpha D_{x}^{+} u_{i, j}^{n}-\lambda \frac{D_{y}^{+} u_{i, j}^{n}}{\left|D_{y} u_{i, j}^{n}\right|_{\beta}}\right)-f_{i, j} .
\end{gathered}
$$

In order to measure the quality of the restored images, we introduce the L2 norm error and

signal to noise ratio(SNR) are defined

$$
\begin{aligned}
& L^{2} \text { norm }=\int_{\Omega}\left(u-u_{0}\right)^{2} d x d y \\
& \text { SNR }=10 \cdot \log _{10}\left(\frac{\int_{\Omega}(f-\bar{f})^{2} d x d y}{\int_{\Omega}(\eta-\bar{\eta})^{2} d x d y}\right), \\
& \text { Where }|\Omega|=\frac{1}{|\Omega|} \int_{\Omega} d x d y, \bar{f}=\frac{1}{|\Omega|} \int_{\Omega} f d x d y \text { and } \bar{\eta}=\frac{1}{|\Omega|} \int_{\Omega} \eta d x d y \text {.The lower }
\end{aligned}
$$
$L^{2}-$ norm error

and the higher the SNR imply that the better the quality of the restoration. All examples were programmed in Matlab 7.4 and use the $2.20 \mathrm{GHz}$ Pentium 4 Windows XP system.

Example 3.1. We first test on the original Lena image shown in Figure 3.1 of size $256 \times 256$. We expose the original image with the additional random Gaussian white noise whose mean variance is 20 . For the ROF model and the modified ROF model, the parameter $\lambda=31.25, \alpha=10^{-5}, \delta=10^{-4}$, the times of iteration is 500 and the time step $t=0.2$. Before processing, the original image with $\mathrm{SNR}=26.0056$ is shown in Figure 3.1. For the restoration image by the ROF model is shown with $\mathrm{SNR}=30.9156$ in Figure 3.1(c). Figure 3.1(d) shows the restoration image generated by our modified model and the SNR $=31.4199$. In order to better understand the image, we also draw the contour map of four related images shown in Figure 3.1(e)-(h). From the contour maps, it's obviously that the ROF model and modified model have good restoration. However, comparing with the image shown in Figure 3.1(g), we can find that the image recovered by the modified model has least staircase effects than the image generated the ROF model shown in Figure 3.1(g). Furthermore, the images shown in Figure 3.1(d) 
looks more natural, especially the right upper side of the region and center region. Simultaneously, we compare the $\mathrm{L}^{2}$ norm error of the noise image, the restoration image and our restoration image. The $\mathrm{L}^{2}$ norm of noise image

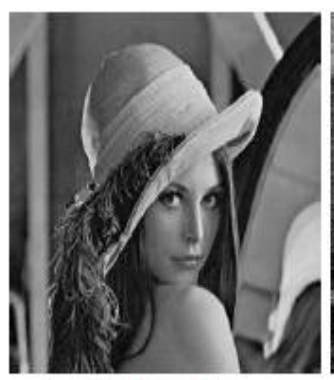

(a).Original image

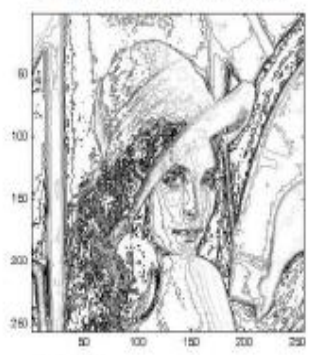

(e).Original image

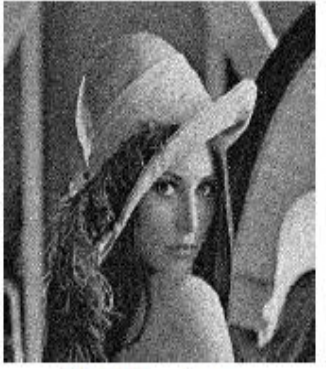

(b).Noisy image

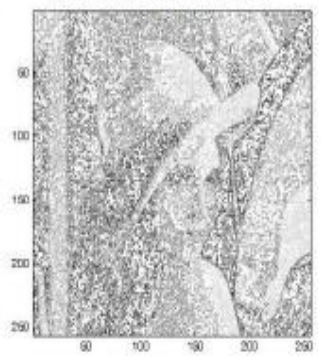

(f).Noisy image

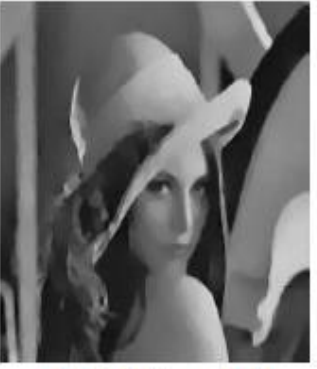

(c).ROF model

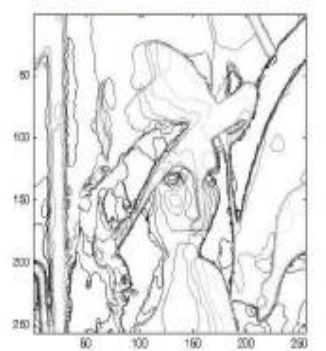

(g).ROF model

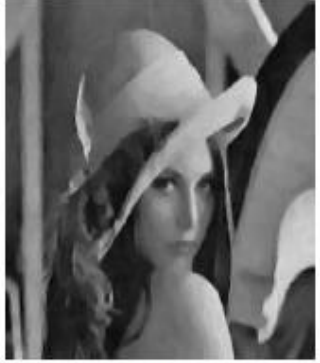

(d).Modified model

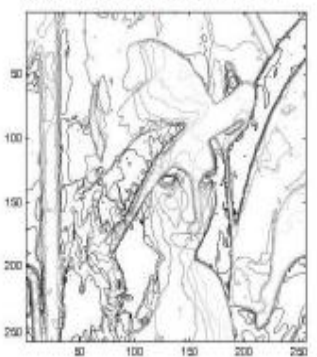

(h).Modified model

Figure 3.1: The related images in Example 3.1. The upper row shows the restored images and the lower row shows the contours related to the upper images generated by the ROF model is $2.6375 \times 10^{7}$. The $\mathrm{L}^{2}$ norm of the restoration image generated by the ROF model and our restoration image are $8.5153 \times 10^{6}$ and $7.5818 \times 10^{6}$, respectively. It is easy to find that the later has better image restoration.

Example 3.2. We test on a MRI image shown in Figure 3.2(a) of size $256 \times 256$. The noise image shown in Figure 3.2(b) is added to the white Gaussian noise with the mean variance as 15 . So the SNR of noise image is 32.7327 and $\mathrm{L}^{2}$ norm error is $1.4716 \times 10^{7}$. We use the same parameters except for the iterative times is 1000 . The restoration image by the ROF model and our modified model are respectively shown in Figure 3.2(c) -(d). The SNR of the restored images shown in Figure 3.2(c)-(d) are 34.6243 and 35.2028. The $\mathrm{L}^{2}$ norm of them are $9.5197 \times 10^{6}$ and $8.3324 \times 10^{6}$, respectively. To really visualize different performance of the schemes only a small portion of the images is depicted in Figure 3.2(e)-(h). By the above facts, we can obtain the same conclusions as restoring the Lena image. 


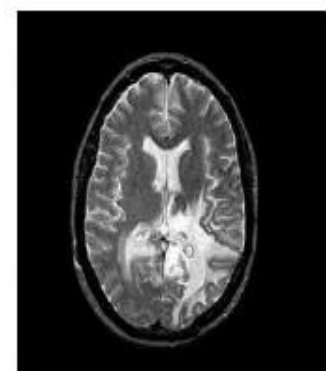

(a).Original image

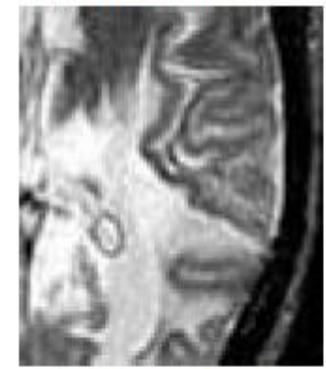

(a1).Original image

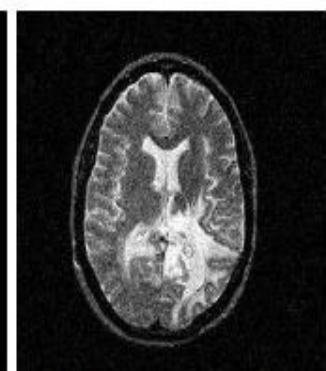

(b).Noisy image

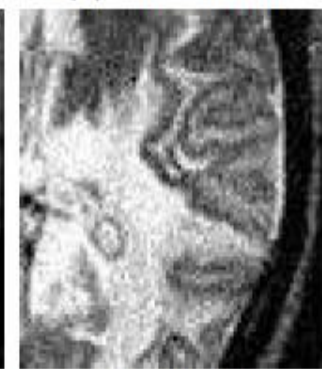

(b1).Noisy image

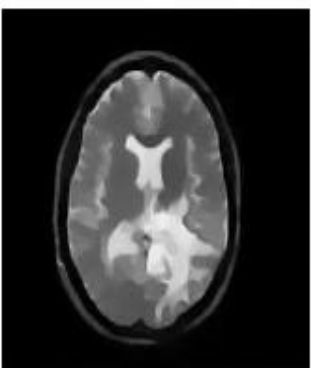

(c).ROF model

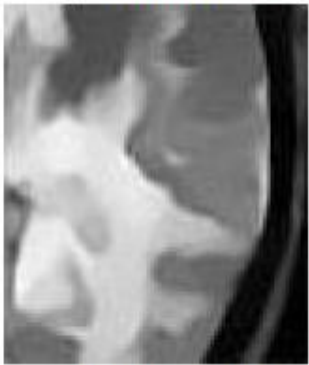

(c1).ROF Model

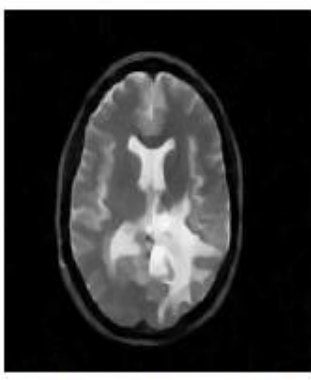

(d).Modified model

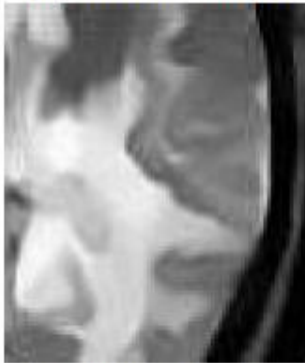

(d1).Modified Model

Figure 3.2: The related images in Example 3.1.

\section{Conclusion}

In this paper, we propose a modified model for the image denoising problem based on the ROF model. The existence and uniqueness of solution and optimization condition for the proposed model are proved. Some numerical examples illustrate the effectiveness of the modified model. Comparing with the method in [3], our proposed model is more natural. In the future, we plan to study some improved algorithms to solve our proposed model.

\section{References}

[1]. C. R. Vogel. Computational Methods for Inverse Problems. Frontiers Appl. Math. 23, SIAM,

Philadelphia, 2002.

[2].T. F. Chan and J. Shen. Image Processing and Analysis: variational, PDE, wavelet, and stochastic

methods. SIAM Publisher, Philadelphia, 2005.

[3].L. Rudin, S. Osher, and E. Fatemi. Nolinear total variation based noise removal 
algorithms. Physica

D 60(1992) 259-268.

[4].E. Giusti. Minimal surfaces and functions of bounded variation. Birkhauser, Boston, 1984.

[5].A. Chambolle and P.L. Lions. Image recovery via total variation minimization and related problems.

Numer. Math. 76(1997), 167-188.

[6].T. F. Chan, A. Marquina, and P. Mulet. High-order total variation-based image restoration. SIAM J.

Sci. Comput. 22(2)(2000) 503-516.

[7].Y.-L. You and M. Kaveh. Fourth-order partial differential equation for noise removal. IEEE Trans.

Image Process. 9(10)(2000) 1723-1730.

[8].M. Lysaker, A. Lundervold, and X.-C. Tai. Noise removal using fourth-order partial differential

equation with applications to medical magnetic resonance images in space and time. IEEE Trans.

Image Process. 12(12)(2003) 1579-1590.

[9].T. F. Chan and S. Esedoglu. Aspects of total variation regularized L1 function approximation. UCLA

CAM Report 04-07 (2004).

[10].M. Lysaker and X. Tai. Iterative image restoration combining total variation minimization and a

second order functional. International Journal of Computer Vision, 66(1):5-18, 2006.

[11].B. Shi, Z. Pang, and Y. Yang. Image restoration based on the hybrid total-variation-type model.

Abstract and applied analysis, 2012. (Doi: 10.1155/2012/376802) 